\title{
Strategie digitali e di progetto per un patrimonio costruito e il suo paesaggio nel sistema di fortificazione del Nord Sardegna
}

Project and digital strategies for a built heritage and its landscape in the coastal fortifications system of Northern Sardinia

\section{Sofia Pieri}

Università degli Studi di Firenze, Florence, Italy, sofia.pieri@unifi.it

\begin{abstract}
The research will deal with new methods about project and digital strategies: first starting from digital survey, operated with 3D laser scanner and photogrammetry procedures, allowing, through various operations, coordinated in a workflow to obtain a single point-cloud, derived from the alignment of all scan, to generate a complex 3D model, so called Building Information Model for Heritage (BIM-H).

The present research project aims to investigate and systematize, the new digital restitution strategies of the fortification existing through laser scanning and digital mapping, in particular, on military structures, as well as reflect on the problem of protection and managing this specific kind of built heritage today so far from the motivations that once determined its construction.

Choosing two case studies, belonging to the coastal fortifications system of Northern Sardinia, built in the same historical period, Forte Cappellini and Punta Rossa, analyze the common constructive characters, the relationship with nature and their current state of conservation, going to verify the possibility of sharing methodologies and strategies of appropriate project.

Passing from an isolated and static vision of work files to one that will be shared and dynamic, it will be possible to offer "food for thoughts" in various areas and disciplines related to Architecture, which will be able to interface simultaneously and work in progress, enriching its content and know-how.

The outcome of the study will allow to recognize the emergence of a new strategy of scientific investigation and design able to positively influence the protection and conservation of military architecture of our peninsula, suggesting theoretical and operational guidelines of different nature, often in shorter times and with results almost unimaginable before, allowing for further analysis and developments the same model in future.
\end{abstract}

Keywords: Fortification, BIM-Heritage, strategies, complexity.

\section{Introduzione}

La fortificazione costiera, come strumento volto a difendere il territorio, è attestata anche nel nord della Sardegna fin dall'epoca spagnola, ma conosce la sua fase più significativa durante il Regno d'Italia. Sin dalla costituzione del nuovo stato era stato posto l'arduo problema di salva- guardare le coste, ma poiché non è possibile fortificarle in modo continuo è stata necessaria una scelta strategica, consistente in una flotta potente con le basi di supporto necessarie. Ed è proprio in questa prospettiva che alla fine del diciannovesimo secolo nacque l'unica roccaforte sarda 
dell'età contemporanea, che, data la sua posizione, era fondamentale per la difesa del confine marittimo occidentale. Doveva infatti fornire assistenza alla flotta che aveva la sua base operativa nelle Bocche di Bonifacio per intervenire rapidamente e contrastare eventuali attacchi francesi sulla costa tirrenica della penisola. A partire dal 1886, l'arcipelago divenne un grande cantiere e nel corso di otto anni prese forma dal nulla una base navale ben attrezzata e convenientemente difesa. La piazza militare poteva essere considerata pienamente efficiente nel 1893, quando il re Umberto I decretò l'istituzione del comando militare marittimo autonomo.

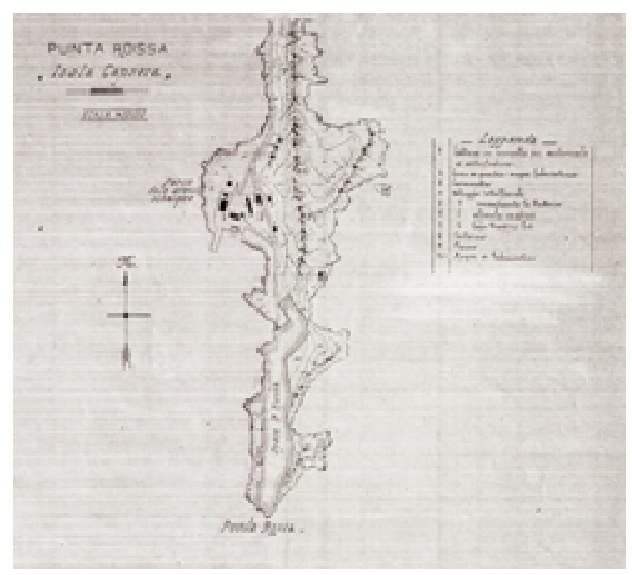

Fig. 1. Planimetria di Punta Rossa, 1922 (AS Genio Militare di La Maddalena).

Le strutture difensive della Maddalena non sono attribuibili alla categoria dei forti, ma piuttosto alle batterie fortificate, poiché sono state progettate e costruite secondo le regole per la costruzione di postazioni di artiglieria da proteggere dai colpi di stato. In base alle caratteristiche, possono essere suddivisi in Opere Basse, per difendere i punti di accesso dagli ancoraggi e Opere Alte, destinati a interdizioni distanti e contrastare i tentativi di sbarco. Punta Rossa appartiene alle opere basse che insieme a Capo Tre Monti controllano il Passo del Levante, mentre Nido d'Aquila e Punta Tegge furono costruite per proteggere il Passo di Ponente. L'elemento caratterizzante delle Opere Basse è quello di essere ben nascoste nel terreno per evitare la localizzazione dal mare: l'armamento costituito dai cannoni a "scomparsa" risponde alla stessa logica. Dovendo così armonizzarsi con la natura dei luoghi, le due fortificazioni di Levante si sviluppano longitudinalmente con connessioni in galleria come Punta Rossa, mentre quelle occidentali come Punta Tegge formano una massa rocciosa costiera. (Belli E. Art. Tratto da In Labore Ingenium, pp. 183-190).

\subsection{Punta Rossa}

Punta Rossa, una lunga appendice sul lato meridionale dell'arcipelago della Maddalena, costituisce il crocevia strategico del mediterraneo da quando fu edificata nel 1887 la Batteria di Punta Rossa dalla Marina del Regno di Sardegna. Insieme alle altre due fortezze di Caprera, Arbuticci e Poggio Rasu, è interamente costruita a mano con blocchi di granito e calcestruzzo. Successivamente dalla Marina Italiana, per difendersi dagli attacchi aerei furono costruite basi periferiche caratterizzate da un assoluto mimetismo. Così anche all'interno dell'area di Punta Rossa c'è un tentativo di ammodernamento tra la I e la II Guerra Mondiale con il riarmo della stazione torpedini e la sostituzione degli armamenti. Inoltre, vi sono diverse strutture come casermette ed altre piccole infrastrutture militari ora in disuso. In definitiva queste opere militari sono di singolare interesse non solo perché esprimono chiaramente i contenuti dei parametri funzionali ma, soprattutto, per il loro aspetto imponente, l'inserimento nella natura, la giustapposizione di elementi murari di contenimento e di sostegno, costituiscono altrettanti spunti e fermenti che si rivelano all'esterno. La logica della difesa ha quindi determinato la morfologia e la distribuzione sul terreno dell'architettura fortificata, obbligando l'architetto e poi l'ingegnere militare a tralasciare tutti gli aspetti generalmente presenti in ogni altra opera edilizia e ad elaborare strumenti e metodi di sintesi tecnologica in grado di adattarsi alla morfologia del luogo. Allo stesso modo è stata sperimentata una metodologia di rilievo e di progetto in grado di adattarsi a tale complesso monumentale sviluppatosi sopra e sottoterra per quasi $1 \mathrm{~km}$ di costa. Il Comprensorio di Punta Rossa si configura come una sorta di penisola irregolare dalla forma di un triangolo, posto all'estremo sud dell'isola di Caprera; 
confina con il mare nei lati ovest, est e sud, è collegato all'isola di Caprera da un istmo di circa $100 \mathrm{~m}$ a nord-ovest dal 1890. L'intera area demaniale è costituita da terreno irregolare a tratti roccioso, si presenta alquanto accidentata e caratterizzata da vegetazione ricca di macchia mediterranea, cisti, ginepro e olivastro. La ex batteria è ubicata all'estremo sud, costituita da fabbricati ad un piano fuori terra con pietre locali ben mimetizzate. Sono presenti nel sottosuolo gallerie che consentono il collegamento tra il Fanale, la Stazione di Vedetta e la batteria. Il Fanale di Punta Rossa è ubicato presso l'estremità dell'opera e viene utilizzato per i fini istituzionali legati alla regolamentazione della navigazione

\subsection{Inserimento paesaggistico}

Vista dal mare, l'Opera Punta Rossa, appare come un susseguirsi disteso di opere murarie che si mimetizzano con le rocce del luogo, dissimulando la loro imponente presenza. Allo stesso modo nella vista da terra del complesso princi- pale gli stessi elementi non sono percepibili nella dimensione reale in quanto distribuiti linearmente su più di cinquecento metri di promontorio con alcuni collegamenti in galleria tra $i$ vari livelli. Le architetture militari si mascherano ed allo stesso tempo cercano visuali funzionali al controllo del territorio, con un'imponenza che solo nei forti nel Nord della Sardegna è dato di vedere e percepire. tramite il caso di Punta Rossa, le linee guida che possano essere spunto anche per altre fortificazioni dell'arcipelago. Partendo dalla propria vocazione e tipologia, come sistema di difesa, in cui le opere basse e le opere alte sono state create per la difesa del Canale di Levante e di Ponente, si cerca non solo di conservarne il patrimonio ma di trasmettere ai posteri la loro storia e logica costruttiva.

\section{Forte Cappellini (già Opera Tre Monti)}

Il Forte è costituito sull'acrocoro di un'emergenza granitica, con precisa volontà di funzione e forma; anche la roccia granitica sottostante e soprastante il forte costituisce perciò parte

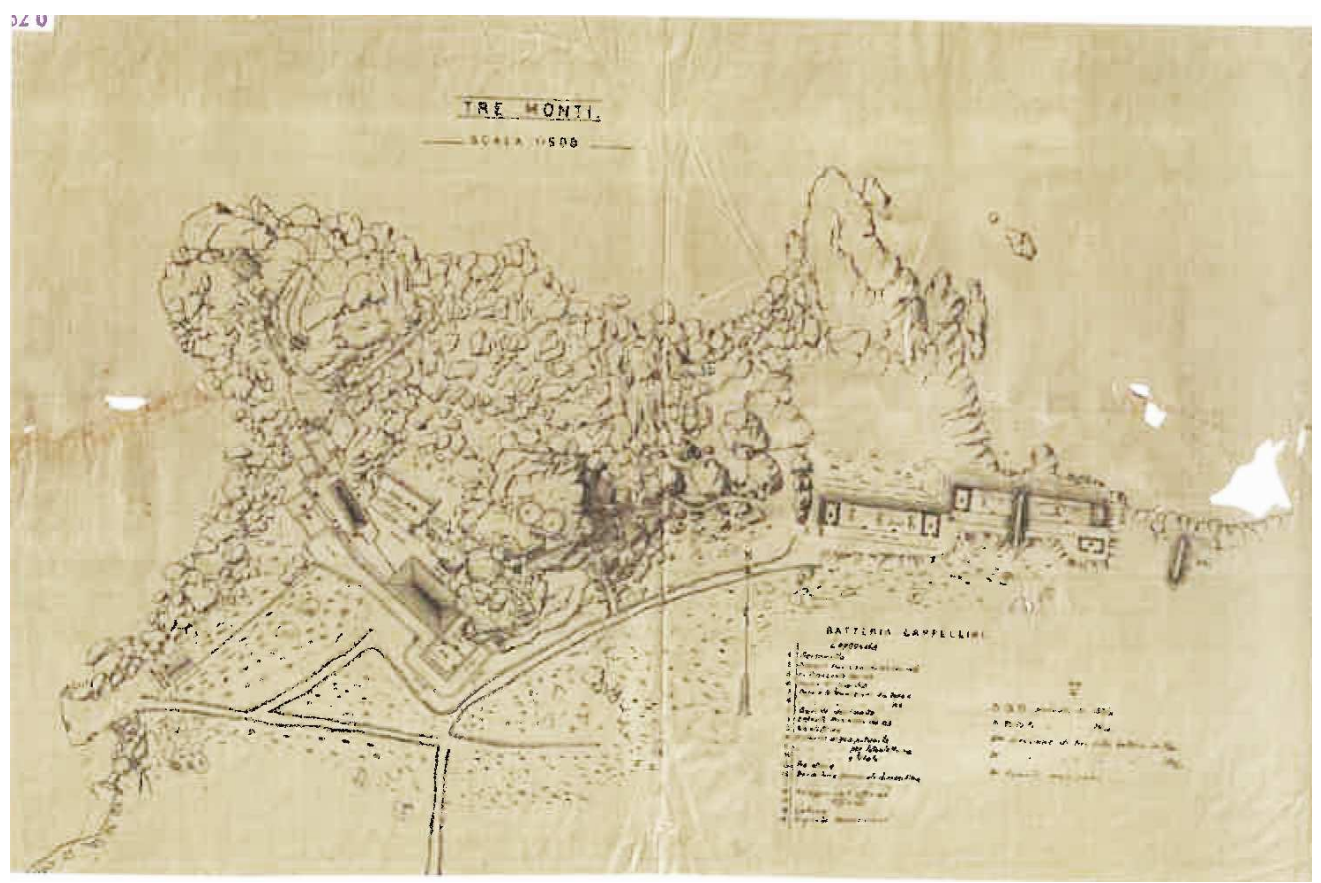

Fig. 2. Documento storico, Opera tre Monti, Baja Sardinia, Sardegna (1891) Archivio del Genio Militare di La Maddalena. 
integrante della struttura costituendone la muraglia difensiva naturale, nonché l'area fino al mare costituisce la sua storica e strutturale pertinenza. E' realizzato con sistemi legati alla tradiizone fortilizia napoleonica, come altri forti dello stesso periodo, dovuto alle immutate tecniche militari. La struttura denominata Forte Cappellini, già opera Tre Monti, è descritta nel suo impianto generale, compreso il piccolo molo, in una tavoletta del Genio Militare datata 1891.

L'attuale consistenza militare dell'area si deve anche ad ulteriori interventi databili a metà degli anni 'Venti dello scorso secolo. L'Opera Tre Monti, edificata mimeticamente tra le rocce dell'omonimo Capo, fu parte del complesso difensivo del Nord Sardegna (da Palau, La Maddalena-Caprera ad Arzechena), realizzato sul finire dell'Ottocento ed in stato di allerta durante il corso della Grande Guerra e della Seconda Guerra Mondiale. E' stata interessata da un potenziamento di armamenti negli anni 'Venti e nel 1943, con l'installazione di postazioni limintrofe antiaeree e antinave.

Il sedime monumentale comprende infatti la batteria fortificata e gli spazi pertinenziali ad essa connessi sia per funzione che per allocazione ad altri manufatti ad essa strumentali.

Le fonti uniscono le vicende dell'Opera Tre Monti a quelle della Piazza Marittima di La Maddalena, disposta a partire dagli anni 'Ottanta del XIX secolo. Dagli anni 'Settanta del XIX all'inizio della I Guerra Mondiale, la Francia aveva rappresentato il più probabile avversario del Regno d'Italia e pertanto durante questa lunga stagione il litorale settentrionale dell'Isola era stato oggetto di un particolare interesse strategico, sia a lungo raggio che nel particolare della sorveglianza e dell'offensiva attraverso le bocche di Bonifacio...

Il rovesciamento delle alleanze rese però vani gli studi e gli investimenti fatti in vista di una guerra navale nel Mediterraneo Occidentale. Durante la Grande Guerra la Piazza de la Maddalena fu spogliata di gran parte dei suoi armamenti per supplire alla carenza di artiglieria pesante del Regio Esercito; così oltre al disarmo parziale delle batterie dell'Arcipelago, tutti i cannoni e tutte le mitragliatrici delle batterie a terra furono indirizzate al fronte austro-ungarico nel Nord Italia. Gli stessi piani militari tornano presto in auge tra il 1919 e il 1940 visto il nuovo peggioramento delle relazioni tra le due potenze; tuttavia le mutate tecniche belliche, con l'introduzione dell'aereo e del sommergibile, resero necessari aggiornamenti tattici e strutturali. Negli Anni 'Venti infatti lo Stato Maggiore della Marina si era fatto promotore del cosiddetto 'Sistema Maddalena-Terranova', (l'attuale Olbia), un progetto teso all'ammodernamento della difesa dell'Arcipelago che prevedeva anche il rafforzamento dello sbarramento a mare; progetto poi nuovamente ridimensionato dal 1924. La Piazzaforte venne infine disarmata negli anni '50 in ottemperanza alle clausole del trattato di pace del ' 47.

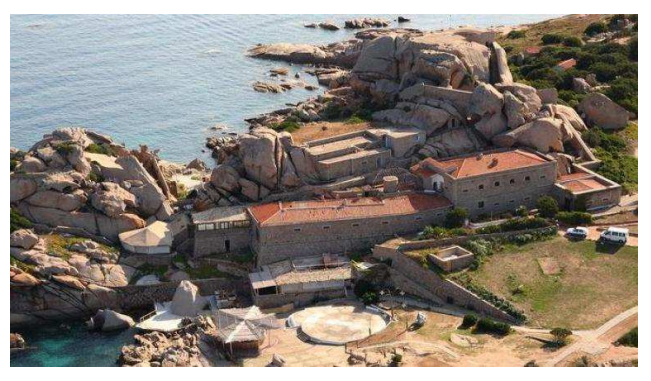

Fig. 3. Foto dall'alto, Forte Cappellini, Baja Sardinia, Sardegna (2016).

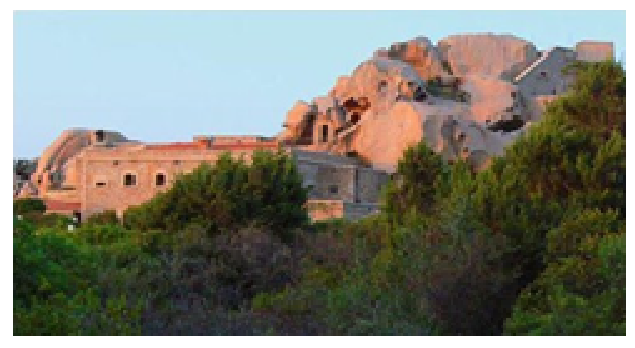

Fig. 4. Foto, Forte Cappellini, Baja Sardinia, Sardegna (2016).

La realizzazione del primo impianto dell'Opera tre Monti, che corrisponde all'attuale sedime e a gran parte delle strutture in esso è da datarsi anteriormente al 1891, data della Tavoletta del Genio Militare che ne rappresenta le fattezze compiute, ma fu certamente ultimata entro il 1889 
giacchè tutte le Opere a terra della Piazza maddalenina risultavano già armate.

\section{Forte Cappellini (già Opera Tre Monti)}

La constante nell'utilizzo del granito locale tende ad assimilare a-cronologicamente il forte alla tipologia meglio rappresentata degli esempi di Palau e dei numerosi dell'arcipelago maddalenino, non considerando tuttavia che questi ultimi mostrano una notevole varietà di articolazione cronologica.

Nell'elenco che seguì allo studio formulato dalla commissione del Comitato di Stato Maggiore Generale per la difesa delle coste. 1886, tra le opere per la realizzazione della Piazza Marittima era compresa anche la Batteria Tre Monti.

Oltre alle opere a terra il sito comprendeva anche lo sbarramento esterno del canale di Levante, attraverso il posizionamento subacqueo di un triplo ordine di mine a circuito, da Punta Rossa a Punta Tre Monti, già portato a termine nel 1884 . Per lo sbarramento, parte del complesso dispositivo subacqueo che interessava tutta la Piazza marittima, erano necessari svariati $\mathrm{km}$ di cavi armati con l'impiego di di particolari natanti e palombari per il posizionamento delle mine, torpedini e gimnoti; per consentire il transito delle imbarcazioni civili nazionali la corrente elettrica del circuito veniva interrotta in corrispondenza dei due varchi posti all'altezza della secca Tre Monti e di Punta Sardegna.

La distanza tra Punta Rossa e Forte Cappellini è infatti di soli $3 \mathrm{~km}, 1,72$ miglia nautiche.

Nel dicembre 1887, nonostante la riduzione di fondi che limitò la realizzazione contemporanea della Piazza, i lavori delle opere finanziate partirono. La realizzazione dell'Opera batteria Tre Monti può dunque essere collocata tra il 1887 e il 1889 , un tempo molto breve che dovette comportare un notevole impiego di forza lavoro. Il primo armamento dell'Opera era di medio calibro con cannoni a scomparsa e mitragliere, come mostra la Legenda della Tavoletta del Genio, 1891 contenuta nell'Archivio del Genio Militare La Maddalena.

Durante i primi anni 'Venti, insieme alle altre Opere Basse, anche la Tre Monti fu riarmata con cannoni Amstrong da 152/40 a tiro rapido, il cui impianto dovette comportare la colmata dei pozzi in ca.

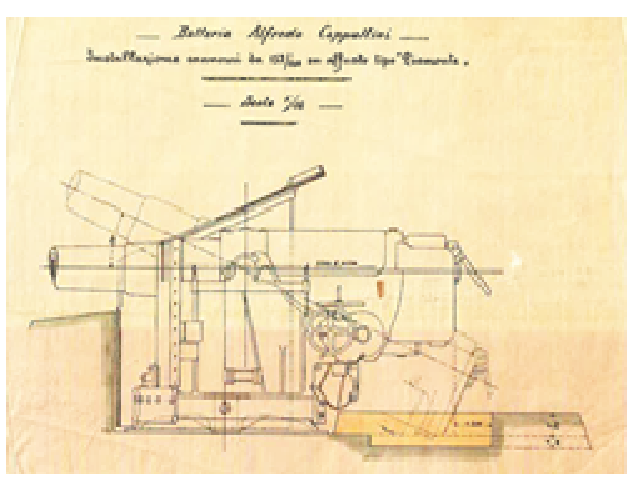

Fig. 5. Documento storico dei cannoni del Forte Cappellini, Baja Sardinia, Sardegna (1890), Archivio del Genio Militare di La Maddalena.

\section{Strategia metodologica}

La ricerca affronta un nuovo metodo di conoscenza e una nuova strategia di progetto: il primo, a partire da rilievo fotografico e dai documenti storici, si basa sulla conoscenza del manufatto cronologica e geometrica, tramite una sturumentazione sempre più avanzata, laserscan $3 \mathrm{D}$, che ha permesso, attraverso varie operazioni, di ottenere una singola nuvola di punti, cioè l'insieme di tutti i punti rilevati. Le possibilità di questa nuvola sono innumerevoli dalla restituzione virtuale fino alla realtà aumentata.

Il secondo parte dalla finalizzazione in BIM (Building Information Modeling), utile non solo per comprendere il modello e gestire innumerevoli dati e materiali, ma per accelerare la rappresentazione dei manufatti all'interno del consteto e le analisi future.

Prendendo esempio dalla mia tesi di Specializzazione sul caso di Punta Rossa a Caprera (Sardegna) possiamo elencare le seguenti fasi:

1) Rilievo digitale con un alto numero di scnasioni. Software: CAM / 2 FARO 3D FOCUS X330.

2) Allineamento delle scansioni in Autodesk Recap Pro fino ad ottenere un unico file detto point cloud. 
3) Gestione e ottimizzazione dei dati raccolticon alcuni software: Bentley Poin Tools e Autodesk Recap Pro $28 \mathrm{~Gb}$.

4) Modellazione del terreno tramite nuvola di punti per ottenere modello georeferenziato. Software: 3D RESHAPER (creazione di mesh per generare curve di livello).

5) Elaborazione della struttura architettonica in BIM. Software: REVIT 2018 Autodesk (gestione della nuvola di punti all'interno del MODELLO BIM con più fasi di progetto).

6) Restituzione virtuale della modellazione (stato di fatto e di progetto) in VR (virtual reality).

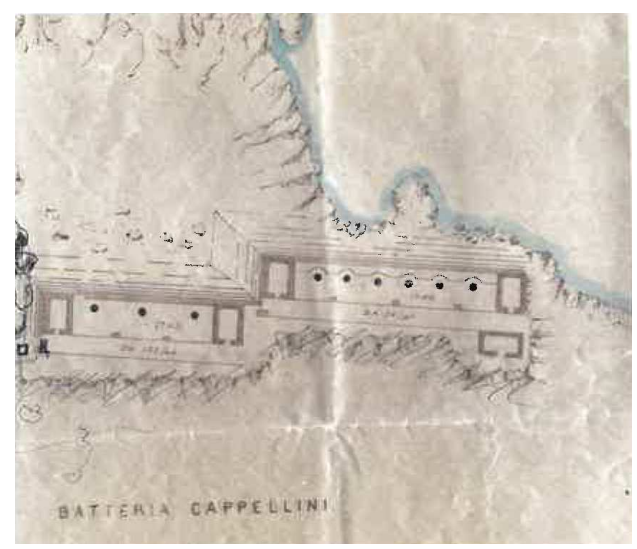

Fig. 6. Planimetria della Batteria Cappellini e della batteria M9, Baja Sardinia, Sardegna (1890), Archivio del Genio Militare di La Maddalena.

\section{Obiettivi}

Il patrimonio storico-militare è immenso e vulnerabile e necessita di opere di salvaguardia, ma non solo, ha bisogno di essere studiato e analizzato in senso critico, per capirne i valori intrinsechi e le potenzialità future. Il fine non è un rilievo o un catalogo, ma un piano per il recupero dell'intero sistema di fortificazioni.

L'abbandono delle strutture dovrebbe trasformarsi in arma attrattiva, come abbandono nella natura, in modo che la guerra rimanga il ricordo nostalgico di quei luoghi.

"Subito dopo l'unita d'Italia e dopo la Seconda guerra mondiale, esaurita la funzione difensiva, i due sistemi di fortificazioni del nostro arcipelago e della prospiciente costa sarda, abbondante e spogliate di tutto ciò che poteva essere utilizzato, hanno perso la loro connotazione di "insieme" un tempo bene identificabile, riducendo la loro presenza a strutture architettoniche isolate e in rovina delle quali resta noto solo il nome, non più il significato".

Il progetto così propone un intervento di restauro e consolidamento conservativo nel caso delle batterie di vedetta di Punta Rossa e di musealizzazione e rifunzionalizzazione di Capo Tre Monti, in cui la caratterizzazione di base dei materiali diventa indispensabile per affrontare la compatibilità chimico-fisica tra $\mathrm{i}$ prodotti consolidanti $\mathrm{e}$ protettivi e i materiali litoidali (pietra e malte). L'intento finale è quello di creare un itinerario museale a cielo aperto, una sorta di via delle fortificazioni.

Nel rispetto di quei capisaldi militari "osservare senza essere osservato" e mimetizzarsi che animano la strategia difensiva dei siti e che rimangono oggi come valori e chiave di lettura indispensabile per comprendere le ragioni dell' insediamento. Il percorso partirà da questi principi fondanti per guidare il visitatore all'interno delle batterie e dei depositi munizioni, fino alle piazzole di tiro da cui sarà possibile osservare l'intero paesaggio, dominando il tratto di mare protetto.

L'ultima linea guida per lo sviluppo di questa via fortificata, sarà la ricostituzione di un legame visivo e strategico con Poggio Rasu-Punta Rossa e Capo Tre Monti, oggi detto Forte Cappellini, in grado di ripristinare la percezione della rete strategica, oggi prevalentemente frammentata.

Da alcuni esempi di interventi, come il caso di Forte Arbuticci oggi Museo Garibaldi, proviamo ad aumentare la consapevolezza degli interventi volti a migliorare e preservare le fortificazioni, cercando di mantenere evidenti le loro caratteristiche identitarie, mimetiche e di rifugio.

\section{Conclusioni}

La collaborazione tra Università ed enti locali potrebbe permettere lo sviluppo di una strategia multidisciplinare applicabile a 2 o più progetti 
paralleli per sviluppare una mappatura completa del patrimonio utile per la tutela e valorizzazione in chiave contemporanea e in base alle necessità espresse dall' amministrazione.

Un primo progetto nato dalla collaborazione tra la Facoltà di Architettura e il Parco con il supporto della Regione, si impegna a rilevare tutto il complesso di Punta Rossa in BIM, per non solo di restauro ma di rifunzionalizzazione, utille per il futuro. e giungere ad un risultato concreto e innovativo delle aree d'intervento analizzate.

Consapevoli che è più facile restaurare dov'era com'era, che sforzarsi a trovare una nuova vita a tutte quelle strutture, vorrei fissare il concetto, che il restauro fa rivivere un 'opera non cancellando i segni del tempo, di cui lei stessa è testimone.

\section{Bibliography}

Battaino. (2006). Forti-Architettura e progetti, Nicolodi, Trento.

Cianchetti, P. (1989). L'isola della Maddalena, documenti e appunti storici II ${ }^{\circ}$, Marisardegna Ed., La Maddalena.

Cianfarani, F. (2013). "Le piscine delle Maree di Alvaro Siza", rivista Hortus, 64.

Comune di La Maddalena (1994). I Forti dell'Arcipelago, P. Sorba Ed., La Maddalena.

Italia Nostra (1987). Mostra sulle fortificazioni dell'estuario di La Maddalena, Rossi Ed., La Maddalena.

Michelini, A. (1863). Storia della Marina Militare del cessato Regno di Sardegna dal 1814 sino alla metà del mese di marzo 1861, Eredi Botta Ed., Torino.

Pieri, S. (2017). "L'opera di Punta Rossa (Sardegna, Italia)", in González Avilés, Á.B., ed., FORTMED 2017. Defensive Architecture of the Mediterranean. XV to XVIII Centuries, Publicacions Universitat d'Alacant, Alacant, vol. $\mathrm{VI}$.

Pieri, S. (2018). "Punta Rossa, Caprera. Knowledge and Project strategies for a built heritage and its landscape", in FORTMED 2018. Defensive Architecture of the Mediterranean. XV to XVIII Centuries, Politecnico di Torino, Torino, vol. VII.

Sotgtiu, G.; Sega, A. (2005). Inglesi nell'Arcipelago, Da Nelson alla fine dell'Ottocento, Sorba Ed., La Maddalena.

Urban, G. (2010). Appunti storico-archeologici sull'area di Punta Rossa, Enti locali La Maddalena.

VV.AA. (1908). Lo sviluppo marittimo del secolo XIX, Leg. Tela Ed., Roma.

VV.AA. (2012). "In Labore Ingenium”, in Pastò, A.M., ed., Atti del convegno di Architettura Militare: centoventidue anni dalla nascita del Genio Militare 1888-2010, Paolo Sorba Ed., La Maddalena. 
